\title{
Electrophysiologic Study-Guided Amiodarone for Sustained Ventricular Tachyarrhythmias Associated With Structural Heart Diseases
}

\author{
Takeshi Aiba, MD; Kenichiro Yamagata, MD; Wataru Shimizu, MD; Atsushi Taguchi, MD; \\ Kazuhiro Satomi, MD; Takashi Noda, MD; Hideo Okamura, MD; Kazuhiro Suyama, MD; \\ Naohiko Aihara, MD; Shiro Kamakura, MD; Takashi Kurita, MD
}

\begin{abstract}
Background Although an electrophysiologic study (EPS) and Holter-monitoring are often helpful in evaluating the efficacy of antiarrhythmic drugs in patients with ventricular tachyarrhythmias (ventricular tachycardia/fibrillation (VT/VF)), the efficacy of EPS- or Holter-guided oral amiodarone therapy in Japanese patients is still unclear.

Methods and Results EPS was performed 1 month after starting amiodarone, and Holter-monitoring was recorded before and 1 month after amiodarone in 188 patients with sustained VT/VF because of structural heart diseases. In spite of the judgment of EPS $(n=89)$ or Holter $(n=75)$, all patients continued amiodarone. Patients were followed up to 3 years and the primary endpoint was VT/VF recurrence and secondary endpoint was death by all cause. Kaplan-Meier estimated the risk of VT/VF recurrence was significantly smaller with EPS-guided amiodarone $(\mathrm{p}<0.01)$ but not with Holter-guided amiodarone. Multivariate Cox hazard analysis revealed that EPS-guided amiodarone was an independent factor suppressing the recurrence of VT/VF $(\mathrm{p}<0.05,95 \%$ confidence interval $=0.15$ to 0.96 ). In the subgroup analysis, EPS-guided amiodarone was effective in patients with relatively well-preserved left ventricular ejection fraction (LVEF $\geq 0.30$ ) but not in patients with lower LVEF (LVEF <0.30).

Conclusion EPS-guided amiodarone was useful for preventing recurrence of VT/VF in patients with a relatively well-preserved LVEF, but not always beneficial in patients with a lower LVEF. (Circ J 2008; 72: 88-93)
\end{abstract}

Key Words: Amiodarone; Electrophysiologic study; Holter monitoring; Ventricular fibrillation; Ventricular tachycardia

V entricular tachyarrhythmias are critically important in the prognosis of patients with structural heart diseases. Amiodarone is one of the most advocated antiarrhythmic drugs available for preventing the recurrence of ventricular tachycardia (VT), ventricular fibrillation (VF), thereby reducing total mortality in patients with VT/VF ${ }^{1-4}$ Although an electrophysiologic study (EPS) and Holter monitoring are performed to evaluate the efficacy of antiarrhythmic drugs, oral amiodarone is often prescribed empirically because the antiarrhythmic effect as guided by EPS or Holter monitoring is controversial! ${ }^{5-9}$ Recent clinical trials have shown that an implantable cardioverter defibrillator (ICD) is clearly superior to amiodarone for preventing sudden arrhythmic death ${ }^{10-13}$ but cannot prevent the recurrence of VT/VF and sometimes gives an intolerable shock to the patient. Therefore, it is still important to clarify how to optimize amiodarone and/or ICD therapies in patients with sustained VT/VF and structural heart diseases!4,15

On the other hand, patients with a lower left ventricular

(Received November 2, 2006; revised manuscript received August 21, 2007; accepted September 13, 2007)

Division of Cardiology, Department of Internal Medicine, National Cardiovascular Center, Suita, Japan

Mailing address: Takashi Kurita, MD, Division of Cardiology, Department of Internal Medicine, National Cardiovascular Center, 5-7-1 Fujishiro-dai, Suita 565-8565, Japan. E-mail: kuritat@ hsp.ncvc.go.jp All rights are reserved to the Japanese Circulation Society. For permissions, please e-mail: cj@j-circ.or.jp ejection fraction (LVEF) derive significantly more benefit from ICD therapy than those with a better preserved LVEF!6-18 Moreover, a recent randomized study reported that amiodarone had no favorable effect on survival but that ICD therapy reduced overall mortality by $23 \%$ in patients with congestive heart failure and LVEF $<35 \% 19$ Therefore, a cardiac function parameter, such as LVEF, is important in determining the prognosis of patients with sustained VT/ VF. The goals of this study were: (1) to evaluate whether or not EPS- or Holter monitoring-guided therapy can stratify the risk of VT/VF recurrence after oral amiodarone, and (2) to investigate the extent to which specific patients subgroups benefit differently from amiodarone therapy.

\section{Methods}

\section{Patients}

This study retrospectively analyzed 400 patients who had been treated with oral amiodarone at the National Cardiovascular Center (Suita, Japan) from 1990 to 2004. All patients had a history of symptomatic sustained VT/VF because of structural heart diseases. We excluded 212 patients with a LVEF $>0.50$, treated with amiodarone for nonsustained VT or atrial arrhythmias, or who had undergone radiofrequency catheter ablation or surgical procedures for VT/VF. Therefore, this study registered 188 patients (mean age, $60 \pm 12$ years; 149 males), which included 77 patients with previous myocardial infarction, 61 with dilated cardio- 
Table 1 Patients' Characteristics

\begin{tabular}{|c|c|c|c|c|}
\hline & \multirow{2}{*}{ Overall } & \multicolumn{2}{|c|}{ EPS-post amiodarone } & \multirow{2}{*}{$\operatorname{EPS}(-)$} \\
\hline & & $V T(-)$ & $V T(+)$ & \\
\hline$n$ & 188 & 27 & 62 & 99 \\
\hline Age (years) & $60 \pm 12$ & $59 \pm 9$ & $58 \pm 11$ & $61 \pm 14$ \\
\hline Sex (male, \%) & $149(79)$ & $22(81)$ & $48(77)$ & $80(81)$ \\
\hline $\operatorname{LVEF}(\%)$ & $30 \pm 12$ & $30 \pm 11$ & $34 \pm 11$ & $28 \pm 12 *$ \\
\hline \multicolumn{5}{|c|}{ Structural heart disease (\%) } \\
\hline Old MI & $77(41)$ & $14(52)$ & $24(39)$ & $39(39)$ \\
\hline$D C M$ & $61(33)$ & $7(26)$ & $18(29)$ & $36(36)$ \\
\hline$H C M$ & $8(4)$ & $0(0)$ & $4(6)$ & $4(4)$ \\
\hline$A R V C$ & $16(9)$ & $1(4)$ & $6(10)$ & $9(9)$ \\
\hline Sarcoidosis & $11(6)$ & $2(7)$ & $6(10)$ & $3(3)$ \\
\hline Valvular heart disease & $12(6)$ & $1(4)$ & $3(5)$ & $8(8)$ \\
\hline Other & $3(1)$ & $2(7)$ & $1(1)$ & $0(0)$ \\
\hline \multicolumn{5}{|l|}{ Presenting arrhythmias (\%) } \\
\hline Sustained VT & $150(80)$ & $23(85)$ & $56(91)$ & $70(71)$ \\
\hline$V F$ & $26(14)$ & $4(15)$ & $2(3)$ & $21(21)$ \\
\hline Sustained VT and VF & $12(6)$ & $0(0)$ & $4(6)$ & $8(8)$ \\
\hline$V F$ total $(\%)$ & $38(20)$ & $4(15)$ & $6(9)$ & $29(29) *$ \\
\hline $\operatorname{ICD}(\%)$ & $81(43)$ & $7(26)$ & $40(65)^{* *}$ & $34(34)$ \\
\hline \multicolumn{5}{|l|}{ Medication (\%) } \\
\hline$A C E I$ & $103(55)$ & $18(67)$ & $30(48)$ & $55(55)$ \\
\hline$\beta$-blocker & $102(55)$ & $12(44)$ & $34(55)$ & $56(57)$ \\
\hline Digitalis & $60(32)$ & $9(33)$ & $13(21)$ & $38(38)$ \\
\hline
\end{tabular}

EPS, electrophysiological study; VT (-), VT or VF is not induced by EPS; VT (+), VT or VF is induced by EPS; LVEF, left ventricular ejection fraction; MI, myocardial infarction; DCM, dilated cardiomyopathy; HCM, hypertrophic cardiomyopathy; ARVC, arrhythmogenic right ventricular cardiomyopathy; VT, ventricular tachycardia; VF, ventricular fibrillation; ICD, implantable cardioverter defibrillator; ACEI, angiotensin converting enzyme inhibitors.

${ }^{*} p<0.05$ vs EPS-VT (+) group; ** $p<0.05$ vs EPS-VT (-) and EPS (-) group.

myopathy, 16 with arrhythmogenic right ventricular cardiomyopathy, 8 with hypertrophic cardiomyopathy, and 11 with cardiac sarcoidosis. The mean LVEF of these patients was $30 \pm 12 \%$ (Table 1 ).

\section{EPS and Holter Monitoring}

After written informed consent was given, EPS was performed in the fasting, nonsedated state before (pre) and 1 month after (post) starting oral amiodarone. All other antiarrhythmic drugs were discontinued. The protocols of the programmed ventricular stimuli have been described in detail previously. In brief, up to 3 premature extrastimuli after an 8-beat stimulus drive were delivered from the right ventricular apex and outflow tract using a quadripolar-electrode catheter, and incremental ventricular stimulation with a constant cycle length. The stimulation protocol was terminated when sustained VT or VF was induced. The efficacy of amiodarone was determined by whether or not a run of VT $>15$ beats could be induced during EPS after starting amiodarone. Thus, we were not concerned about the inducibility of VT/VF before amiodarone therapy.

Twenty-four hours Holter electrocardiogram was recorded on magnetic tape before drug therapy, and repeated 1 month after administration of amiodarone and analyzed by computer to determine the frequency of arrhythmias. The efficacy of amiodarone by Holter recording was assessed by the criteria of the ESVEM trial ${ }^{20}$ First, patients with total premature ventricular contractions (PVC) less than 300/day before amiodarone were excluded from the Holter judgment as an "undetermined" group. Therefore, patients with total PVCs more than $300 /$ day before amiodarone and $70 \%$ reduction in the PVC count, $80 \%$ reduction in the PVC pair count, $90 \%$ reduction in the VT count, and absence of any runs of VT $>15$ beats were defined as "effective", but patients with no response to these criteria were defined as
Table 2 EPS and Holter Judgments

\begin{tabular}{lccc}
\hline \hline & \multicolumn{2}{c}{ EPS post amiodarone } & \multirow{2}{*}{$\begin{array}{c}\text { EPS- } \\
\text { post }(-)\end{array}$} \\
\cline { 2 - 3 } & $V T(-)$ & $V T(+)$ & \\
\hline EPS pre amiodarone & 1 & 0 & 1 \\
$V T(-)(n=2)$ & 3 & 16 & 18 \\
$V T(+)(n=37)$ & 23 & 46 & 80 \\
EPS-pre $(-)(n=149)$ & 27 & 62 & 99 \\
Total $(n=188)$ & & & \\
Holter criteria & 7 & 9 & 21 \\
$\quad$ Effective $(n=37)$ & 7 & 10 & 21 \\
Ineffective $(n=38)$ & 13 & 43 & 57 \\
Undetermined $(n=113)$ & & & \\
\hline
\end{tabular}

EPS-pre (-), EPS before amiodarone is not performed; EPS-post (-), EPS after amiodarone is not performed. Other abbreviations see in Table 1.

"ineffective".

Follow-up After Amiodarone

Whether or not they had EPS or Holter monitoring, all patients continued treatment with amiodarone, the loading dose of which was 300 or $400 \mathrm{mg} /$ day for 2 weeks followed by a maintenance dose of 150 or $200 \mathrm{mg} /$ day. However, amiodarone was discontinued when critical side effects developed or it was obviously ineffective. All patients were followed up to 36 months and the primary endpoint was recurrence of VT/VF and the secondary endpoint was death from all causes. Implantation of an ICD was recommended in patients who were considered to be "ineffective" with amiodarone or had a history of syncope because of VT/VF.

\section{Statistical Analysis}

The continuous variables are expressed as mean \pm SD and were compared by an unpaired t-test when appropriate. Cumulative event rates were calculated by the Kaplan- 


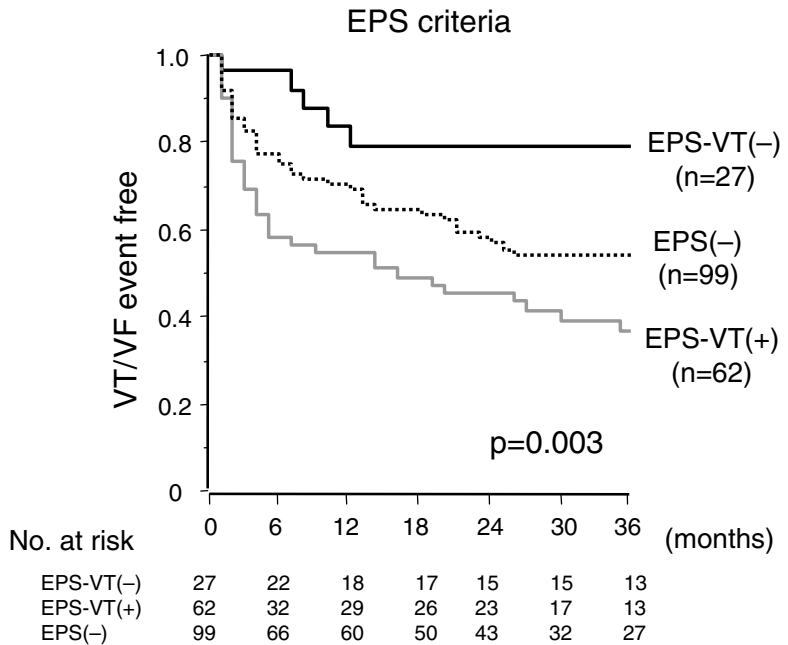

Fig 1. Cumulative risk of recurrent ventricular tachycardia/fibrillation (VT/VF) after amiodarone therapy in patients judged by electrophysiological study (EPS) criteria. EPS stratified the risk of VT/VF recurrence after amiodarone. EPS-VT(+), patients with inducible VT/VF by EPS after amiodarone; EPS-VT(-), patients with no inducible VT/VF by EPS after amiodarone; EPS(-), patients without EPS after amiodarone.

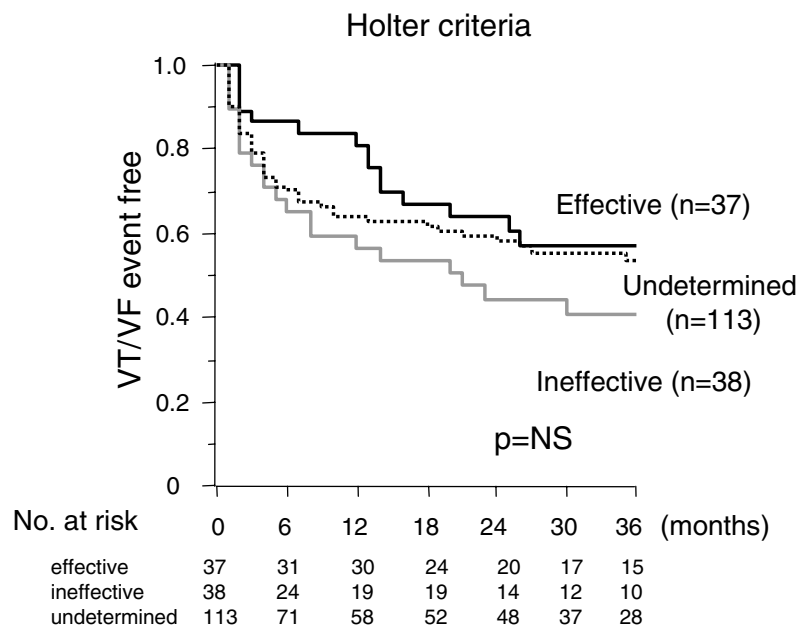

Fig 2. Cumulative risk of recurrent ventricular tachycardia/fibrillation (VT/VF) after amiodarone therapy in patients judged by Holtermonitoring criteria. Holter-monitoring could not stratify the risk of VT/VF recurrence after amiodarone. Effective, patients judged amiodarone effective by Holter; Ineffective, patients judged amiodarone ineffective by Holter; Undetermined, patients excluded from the Holter judgment.

Table 3 Cox Hazard Regression Analysis of VT/VF Recurrence

\begin{tabular}{lcc}
\hline \hline & OR $(95 \%$ CI $)$ & $p$ value \\
\hline Age & $1.26(0.80-1.99)$ & 0.33 \\
Sex (male) & $0.93(0.54-1.60)$ & 0.78 \\
Basal disease (Old MI) & $0.75(0.45-1.25)$ & 0.27 \\
LVEF <30\% & $1.47(0.94-2.32)$ & 0.09 \\
EPS post amiodarone & & \\
$\quad$ VT (+) & $1.71(1.07-2.75)$ & 0.02 \\
$\quad$ VT (-) & $0.34(0.15-0.96)$ & 0.04 \\
Holter judgment & & \\
$\quad$ Ineffective & $1.47(0.87-2.50)$ & 0.15 \\
$\quad$ Effective & $0.77(0.42-1.43)$ & 0.42 \\
\hline
\end{tabular}

Abbreviations as in Tables 1,2.
Meier method. The significance of the difference between treatment groups was assessed with the log-rank test. Cox regression analysis was performed on the patients' baseline characteristics to investigate and compare the influence of different variables. Statistical significance was established as $\mathrm{p}<0.05$.

\section{Results}

\section{EPS and Holter Monitoring}

Table 2 summarizes the results of EPS and Holter monitoring. The EPS before amiodarone was performed in 39 patients, and induced VT/VF in 37 (95\%). The EPS after amiodarone was performed in 89 patients, and could not induce VT/VF in 27 (30\%) patients (EPS-VT(-) group), but still induced VT/VF in $62(70 \%)$ patients (EPS-VT(+) group). The remaining 99 patients taking amiodarone without judgment by EPS were defined as EPS(-) group.

Holter monitoring before and after amiodarone treatment was recorded in 139 patients; however, 64 patients had less PVCs than the Holter evaluation before amiodarone (300/day). Therefore, the remaining 75 patients were judged as amiodarone effective $(n=37)$ or ineffective $(n=38)$ by Holter monitoring.

\section{Follow-up}

During the follow-up period of $23 \pm 13$ (range 1-36) months, $82(44 \%)$ patients had recurrence of VT. Moreover, $28(20 \%)$ patients died during follow-up because of heart failure $(n=8)$, sudden unexpected death $(n=8)$, pneumonia $(\mathrm{n}=2)$, and unknown causes $(\mathrm{n}=10)$. Side-effects of amiodarone occurred in $39(21 \%)$ patients, including hypothyroidism $(n=20)$, proarrhythmia $(n=5)$, pneumonia $(n=$ $11)$, leukocytopenia $(n=1)$, and liver dysfunction $(n=2)$. Amiodarone was discontinued in $13(8 \%)$ patients because of serious side-effects.

Fig 1 illustrates the follow-up results of patients under the EPS criteria. Among those assigned to the EPS-VT(+) group, the rate of VT/VF recurrence was $45.6 \%$ and $63.9 \%$ at 1 and 3 years, respectively. Conversely, in the EPS-VT(-) group it was $21.3 \%$ and $21.3 \%$, and for the EPS(-) group $31.0 \%$ and $46.6 \%$ at 1 and 3 year's follow-up, respectively. Therefore, the VT/VF recurrence risk after amiodarone was significantly lower in the order of EPS-VT(-), EPS(-), and EPS-VT $(+)$ groups $(\mathrm{p}<0.003)$. Table 1 summarizes the clinical characteristics in the 3 groups. Age, sex, basal disease, and medication, except antiarrhythmic drugs, did not differ between them, although LVEF was lower in the EPS $(-)$ group than in the EPS-VT(+) group $(28 \pm 12 \%$ vs $34 \pm 11 \%$; $\mathrm{p}=0.01$ ), and VF incidence before amiodarone was higher in the EPS(-) group than in the EPS-VT(+) group (29\% vs $9 \%$; $\mathrm{p}=0.01$ ). ICDs were consequently implanted in many of the EPS-VT(+) group compared with the EPS-VT(-) and EPS $(-)$ groups $(65 \%$ vs $26 \%, 34 \%$, respectively; $\mathrm{p}<0.05$ ).

Fig 2 illustrates the follow-up results under the Holter criteria. In the patients assigned to the effective group, the VT/VF recurrence rates were $19.1 \%$ and $42.8 \%$ (1 and 3 years, respectively), whereas in the ineffective group, they were $43.4 \%$ and $59.6 \%$ ( 1 and 3 years, respectively) ( $\mathrm{p}=\mathrm{NS}$ ). Therefore, Holter monitoring cannot stratify the risk of VT/VF recurrence after amiodarone.

Table 3 shows the results of multivariate Cox hazard regression analysis for the recurrence of VT/VF after amiodarone. The clinical factors, age, gender, basal disease 

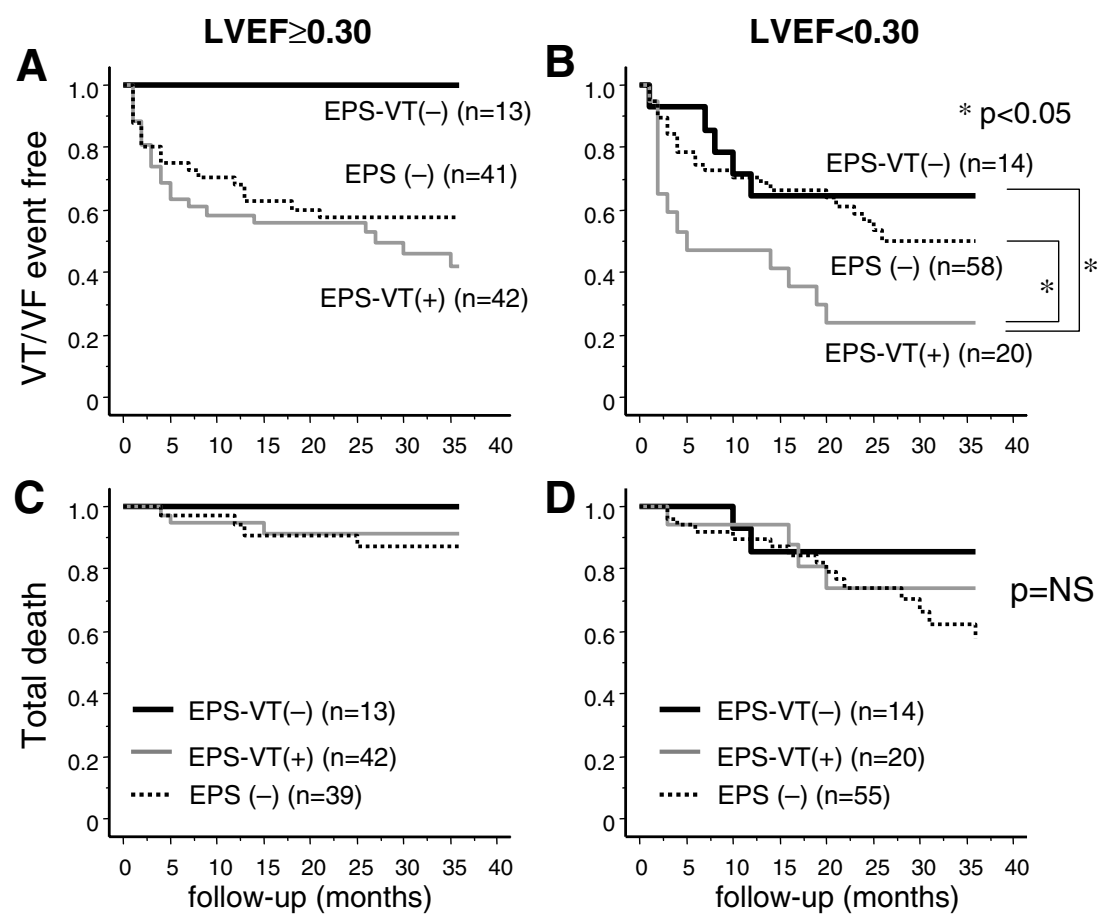

Fig 3. Cumulative risk of recurrent ventricular tachycardia/fibrillation (VT/VF) and total death after amiodarone therapy in different range of left ventricular ejection fraction (LVEF). Electrophysiological study (EPS) judgment could classify the risk of recurrent VT/VF and mortality after amiodarone in patients with $\mathrm{LVEF} \geq 30 \%$ (A and $\mathrm{C}$, respectively) but not in those with LVEF $<30 \%$ (B and $\mathrm{D}$, respectively). EPS-VT(+), patients with inducible VT/VF by EPS after amiodarone; EPS-VT(-), patients with no inducible VT/VF by EPS after amiodarone; EPS(-), patients without EPS after amiodarone.

(myocardial infarction) and Holter judgments were not related to VT/VF recurrence. The independent clinical factors for VT/VF recurrence were inducibility of VT/VF by EPS after amiodarone (odds ratio (OR) $1.71,95 \%$ confidence interval (CI) 1.07-2.75, p=0.02). Lower LVEF $(<30 \%)$ increased the risk of VT/VF recurrence but not significantly (OR 1.47, 95\% CI 0.94-2.32, $\mathrm{p}=0.09$ ).

\section{EPS-Guided AmiodaroneTherapy and LVEF}

Because of the possibility of lower LVEF increasing risk of VT/VF recurrence after amiodarone, we evaluated the EPS-guided amiodarone therapy in subgroups. Therefore, among the patients with relatively preserved LVEF $(\geq 30 \%)$ $(n=94)$, there was no VT/VF recurrence in the EPS-VT(-) group, whereas the rates of VT/VF recurrence for the EPSVT(+) group were $42.1 \%$ and $58.5 \%$, and those for the EPS(-) group were $32.3 \%$ and $42.7 \%$ (at 1 and 3 years, respectively) (Fig 3A). Thus, the risk of VT/VF recurrence was significantly lower in the EPS-VT(-) group compared with the EPS-VT(+) and EPS(-) groups. In contrast, among patients with lower LVEF $(<30 \%)(n=91)$, the rates of VT/VF recurrence for the EPS-VT(+) group were significantly higher $(58.6 \%$ and $76.4 \%$ at 1 and 3 years, respectively) than those for the EPS-VT(-) (35.7\% and 35.7\%) and EPS(-) group $(30.0 \%$ and $51.0 \%$, respectively) $(\mathrm{p}<0.05)$, whereas there was no significant difference in the recurrence rate between the EPS-VT(-) and the EPS(-) groups (Fig 3B). Furthermore, in patients with $\mathrm{LVEF} \geq 30 \%$, no patients died in the EPS-VT(-) group, whereas 3 of 42 patients in the EPS-VT(+) group and 4 of 39 patients in the EPS(-) group died during follow-up (Fig 3C). However, in patients with $\mathrm{LVEF}<30 \%, 2$ of 14 patients in the EPS$\mathrm{VT}(-), 4$ of 20 patients in the EPS-VT(+) and 15 of 55 patients in the EPS(-) group died during follow-up ( $p=N S)$ (Fig 3D).

Table 4 shows the sensitivity, specificity, positive predictive value, negative predictive value, and accuracy for recurrence of VT/VF for Holter, EPS pre-post and EPS-post
Table 4 Predictors of Arrhythmic Events

\begin{tabular}{lrrrrc}
\hline \hline & $S N S$ & $S P C$ & $P P V$ & $N P V$ & $A C C$ \\
\hline Holter-judgment $(n=75)$ & 55 & 59 & 58 & 56 & 57 \\
EPS pre-post amiodarone $(n=19)$ & 69 & 100 & 100 & 38 & 74 \\
EPS post amiodarone $(n=89)$ & 59 & 81 & 88 & 46 & 66 \\
LVEF $\geq 30 \%(n=55)$ & 52 & 100 & 100 & 35 & 62 \\
LVEF $<30 \%(n=34)$ & 70 & 64 & 74 & 60 & 68
\end{tabular}

SNS, sensitivity; SPC, specificity; PPV, positive predictive value; NPV, negative predictive value; ACC, accuracy. Other abbreviations as in Table 1 .

amiodarone therapy. The EPS judgment showed lower sensitivity but significantly higher specificity for recurrence of VT/VF, especially in patients with $\mathrm{LVEF} \geq 30 \%$.

\section{ICD and LVEF}

We further analyzed the relationship between ICD and LVEF in patients treated with amiodarone. As shown in Fig 4A, the mortality of patients treated with amiodarone plus ICD did not differ between patients with higher $(\geq 30 \%)$ and lower $(<30 \%)$ LVEF. However, among patients with no ICD (amiodarone only) (Fig 4B), patients with LVEF $\geq 30 \%$ had a similar mortality to those with ICD, but patients with $\mathrm{LVEF}<30 \%$ had a significant worse mortality than the patients with higher LVEF $(p=0.01)$. Therefore, patients with moderate to severe LV dysfunction achieved the greatest benefit from ICD therapy.

\section{Discussions}

\section{Major Findings}

This study retrospectively demonstrated the long-term effect of EPS-guided oral amiodarone therapy in Japanese patients with a history of life-threatening ventricular tachyarrhythmias because of structural heart diseases. EPSguided amiodarone could reduce the recurrence of VT, especially in patients with relatively preserved $(\geq 30 \%)$ 


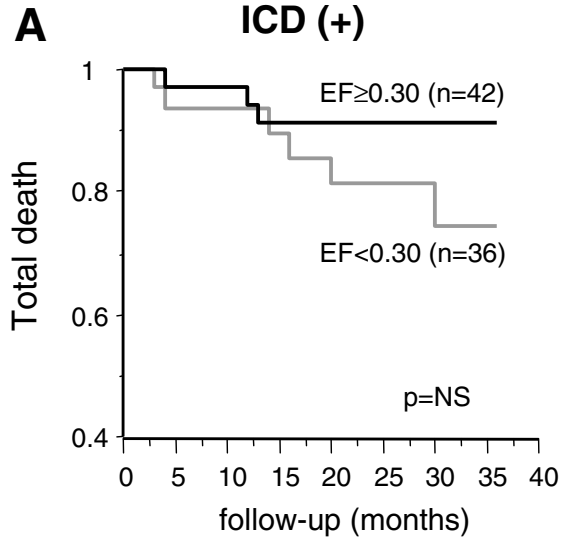

LVEF, but was not always beneficial in patients with lower $(<30 \%)$ LVEF. Therefore, amiodarone-treated patients with lower LVEF but not an implanted ICD remain at higher risk of sudden death.

\section{EPS or Holter-Guided Amiodarone}

Previous studies showed that EP-guided amiodarone therapy was useful for predicting recurrence of VT in patients at high risk for sudden cardiac death5,7,8,21 McGovern et al reported that the sensitivity, specificity and accuracy of EP testing for recurrent VT was $58 \%, 91 \%$ and $67 \%$, respectively.22 Those data coincide with our results showing that EPS-guided therapy has low sensitivity (59\%), but high specificity $(81 \%)$, for recurrence of VT after amiodarone. Moreover, this low sensitivity and high specificity of EP testing is more prominent in patients with LVEF $\geq 30 \%$ (52\% and $100 \%$, respectively) (Table 4 ). In this study, there were a small number of patients available for checking inducibility of VT before amiodarone, but the results of EPSguided amiodarone are similar to those previously reported. Thus, it is not necessary to perform an EPS before starting amiodarone, and patients with LVEF $\geq 30 \%$ and non-inducible VT according to he EPS after amiodarone may remain free from recurrence of $\mathrm{VT}$.

The ESVEM study showed that there was no significant difference between EPS and Holter monitoring in the probability of arrhythmic events occurring after antiarrhythmic drugs $2^{20}$ However, that study mainly examined the effectiveness of class I antiarrhythmic drugs, not amiodarone. The efficacy of amiodarone by Holter monitoring is also controversial. Veltri et al reported that Holter monitoring could predict the long-term efficacy of amiodarone? Nasir et al showed that amiodarone strongly suppressed PVCs but this suppression did not predict clinical outcome ${ }^{23}$ Our finding that a Holter judgment could not predict the recurrence of VT after amiodarone may have resulted because (1) there was a smaller number of patients undergoing Holter monitoring before and after amiodarone, (2) twenty-four hours recording cannot detect the number of PVCs or VT precisely, and (3) the apparent number of PVCs might have no relation to the trigger of critical VT or VF. In this study EPS was clearly superior to Holter monitoring for evaluating amiodarone efficacy, but EPS is invasive and is not always performed in all patients.

\section{Amiodarone, ICD and LVEF}

Zhu et al suggested that EPS testing during amiodarone therapy was useful for predicting arrhythmia recurrence in patients without new or worsening congestive heart failure? Other previous reports suggest that patients with lower LVEF $(<35 \%)$ have a higher incidence of sudden cardiac death after amiodarone! ${ }^{16-18}$ Those results are consisted with our subgroup analysis showing that EPS-guided amiodarone therapy is beneficial for patients with $\mathrm{LVEF} \geq 30 \%$ but not $<30 \%$. Therefore, it is suggested that ICD is indicated in patients with lower LVEF $(\leq 30-35 \%)$ and a history of syncope or sustained VT/VF! $7,24,25$ On the other hand, patients with a relatively preserved LVEF ( $\geq 35 \%$ ) do not always have better survival by ICD compared with amiodarone. 16

In this study, EPS-guided amiodarone responders with a LVEF $\geq 30 \%$ were considered to be lower risk for sudden cardiac death, whereas patients judged as amiodarone nonresponders or with $\mathrm{LVEF}<30 \%$ remain high risk for sudden death. Although our data could not compare between amiodarone and ICD therapy in high-risk patients, amiodaronetreated patients with lower LVEF, but not an implanted ICD, remain at higher risk of sudden death (Fig 4B) and should be considered for additional ICD therapy, as previously reported. ${ }^{4}$

In patients with congestive heart failure and LVEF $<35 \%$, a recent randomized study reported that amiodarone has no favorable effect on survival compared with placebo, but that ICD therapy reduced overall mortality by $23 \% !^{19}$ Although ICD reduces mortality compared with antiarrhythmic drugs, it is estimated that up to $50 \%$ of patients with an ICD ultimately need antiarrhythmic drug therapy to suppress frequent episodes of VT or supraventricular tachyarrhythmias, and that amiodarone is the most commonly used drug for this purpose in Japanese patients ${ }^{15}$ Recently, Connolly et al reported that amiodarone plus $\beta$-blocker was effective for preventing ICD shocks, but increased the risk of drugrelated adverse effect ${ }^{26}$ Therefore, further studies in the Japanese patient population are necessary to evaluate whether or not amiodarone can improve a patient's clinical outcome by reducing the amount of ICD shocks. ${ }^{4}$

\section{Study Limitations}

First, the study was not a prospective evaluation of EPSor Holter-guided amiodarone treatment, so the direct efficacy of EPS or Holter-guided amiodarone in preventing the recurrence of VT/VF was not demonstrated; rather, an excellent prognosis for patients treated with EPS-guided amiodarone, especially in patients with a well-preserved LVEF, was demonstrated. Second, this study compared follow-up results between patients judged as amiodarone responder or non-responder by EPS, but did not compare 
amiodarone responders with control patients. Therefore, it might overestimate the effectiveness of EPS-guided amiodarone therapy for suppression of recurrent VT/VF. Third, this study contained a small number of patients, and a multicenter trial with a large number of patients will be necessary to demonstrate the effect of amiodarone and ICD therapy more accurately in Japanese patients ${ }^{14,15}$ Fourth, this study focused on the risk of recurrent (secondary) VT/VF, not on primary prevention. It is still controversial whether amiodarone and/or ICD are indicated in patients with nonsustained VT and lower LVEF for primary prevention of sudden death.

\section{References}

1. Sim I, McDonald KM, Lavori PW, Norbutas CM, Hlatky MA. Quantitative overview of randomized trials of amiodarone to prevent sudden cardiac death. Circulation 1997; 96: 2823-2829.

2. Connolly SJ. Evidence-based analysis of amiodarone efficacy and safety. Circulation 1999; 100: 2025-2034.

3. Investigators ATM-A. Effect of prophylactic amiodarone on mortality after acute myocardial infarction and in congestive heart failure: Meta-analysis of individual data from 6500 patients in randomised trials. Lancet 1997; 350: 1417-1424.

4. Aiba T, Kurita T, Taguchi A, Shimizu W, Suyama K, Aihara N, et al. Long-term efficacy of empirical chronic amiodarone therapy in patients with sustained ventricular tachyarrhythmia and structural heart disease. Circ J 2002; 66: 367-371.

5. Kadish AH, Buxton AE, Waxman HL, Flores B, Josephson ME, Marchlinski FE. Usefulness of electrophysiologic study to determine the clinical tolerance of arrhythmia recurrences during amiodarone therapy. J Am Coll Cardiol 1987; 10: 90-96.

6. Veltri EP, Reid PR, Platia EV, Griffith LS. Amiodarone in the treatment of life-threatening ventricular tachycardia: Role of Holter monitoring in predicting long-term clinical efficacy. J Am Coll Cardiol 1985; 6: 806-813.

7. Zhu J, Haines DE, Lerman BB, DiMarco JP. Predictors of efficacy of amiodarone and characteristics of recurrence of arrhythmia in patients with sustained ventricular tachycardia and coronary artery disease. Circulation 1987; 76: 802-809.

8. Greenspon AJ, Volosin KJ, Greenberg RM, Jefferies L, Rotmensch $\mathrm{HH}$. Amiodarone therapy: Role of early and late electrophysiologic studies. J Am Coll Cardiol 1988; 11: 117-123.

9. Yuge M, Niwano S, Moriguchi M, Sasaki T, Hirasawa S, Imaki R, et al. Clinical significance of the electrophysiologic study (EPS)-guided therapy for the secondary prevention of ventricular tachycardia. Circ J 2006; 70: 268-272.

10. Buxton AE, Lee KL, Fisher JD, Josephson ME, Prystowsky EN, Hafley G. A randomized study of the prevention of sudden death in patients with coronary artery disease: Multicenter Unsustained Tachycardia Trial Investigators. N Engl J Med 1999; 341: 1882-1890.

11. Connolly SJ, Gent M, Roberts RS, Dorian P, Roy D, Sheldon RS, et al. Canadian implantable defibrillator study (CIDS): A randomized trial of the implantable cardioverter defibrillator against amiodarone. Circulation 2000; 101: 1297-1302.

12. Kuck KH, Cappato R, Siebels J, Ruppel R. Randomized comparison of antiarrhythmic drug therapy with implantable defibrillators in patients resuscitated from cardiac arrest: The Cardiac Arrest Study Hamburg (CASH). Circulation 2000; 102: 748-754.

13. Schlapfer J, Rapp F, Kappenberger L, Fromer M. Electrophysiologi- cally guided amiodarone therapy versus the implantable cardioverterdefibrillator for sustained ventricular tachyarrhythmias after myocardial infarction: Results of long-term follow-up. J Am Coll Cardiol 2002; 39: 1813-1819.

14. Kurita T, Mitamura H, Aizawa Y, Nitta T, Aonuma K, Tsuboi N, et al. Japanese randomized trial for investigation of a combined therapy of amiodarone and implantable cardioverter defibrillator in patients with ventricular tachycardia and fibrillation: The Nippon ICD Plus Pharmacologic Option Necessity study design. Circ J 2006; 70: 316-320.

15. Satomi K, Kurita T, Takatsuki S, Yokoyama Y, Chinushi M, Tsuboi $\mathrm{N}$, et al. Amiodarone therapy in patients implanted with cardioverterdefibrillator for life-threatening ventricular arrhythmias. Circ J 2006; 70: $977-984$.

16. Domanski MJ, Sakseena S, Epstein AE, Hallstrom AP, Brodsky MA, Kim S, et al. Relative effectiveness of the implantable cardioverterdefibrillator and antiarrhythmic drugs in patients with varying degrees of left ventricular dysfunction who have survived malignant ventricular arrhythmias: AVID Investigators: Antiarrhythmics Versus Implantable Defibrillators. J Am Coll Cardiol 1999; 34: 1090-1095.

17. Connolly SJ, Hallstrom AP, Cappato R, Schron EB, Kuck KH, Zipes DP, et al. Meta-analysis of the implantable cardioverter defibrillator secondary prevention trials: AVID, CASH and CIDS studies. Antiarrhythmics vs Implantable Defibrillator study; Cardiac Arrest Study Hamburg; Canadian Implantable Defibrillator Study. Eur Heart J 2000; 21: 2071-2078.

18. Sheldon R, Connolly S, Krahn A, Roberts R, Gent M, Gardner M. Identification of patients most likely to benefit from implantable cardioverter-defibrillator therapy: The Canadian Implantable Defibrillator Study. Circulation 2000; 101: 1660-1664.

19. Bardy GH, Lee KL, Mark DB, Poole JE, Packer DL, Boineau R, et al. Amiodarone or an implantable cardioverter-defibrillator for congestive heart failure. N Engl J Med 2005; 352: 225-237.

20. Mason JW. A comparison of seven antiarrhythmic drugs in patients with ventricular tachyarrhythmias: Electrophysiologic Study versus Electrocardiographic Monitoring Investigators. N Engl J Med 1993; 329: $452-458$

21. Horowitz LN, Greenspan AM, Spielman SR, Webb CR, Morganroth $\mathrm{J}$, Rotmensch $\mathrm{H}$, et al. Usefulness of electrophysiologic testing in evaluation of amiodarone therapy for sustained ventricular tachyarrhythmias associated with coronary heart disease. Am J Cardiol 1985; 55: 367-371.

22. McGovern B, Garan H, Malacoff RF, DiMarco JP, Grant G, Sellers $\mathrm{TD}$, et al. Long-term clinical outcome of ventricular tachycardia or fibrillation treated with amiodarone. Am J Cardiol 1984; 53: $1558-$ 1563.

23. Nasir N Jr, Doyle TK, Wheeler SH, Pacifico A. Usefulness of Holter monitoring in predicting efficacy of amiodarone therapy for sustained ventricular tachycardia associated with coronary artery disease. Am J Cardiol 1994; 73: 554-558.

24. Olson PJ, Woelfel A, Simpson RJ Jr, Foster JR. Stratification of sudden death risk in patients receiving long-term amiodarone treatment for sustained ventricular tachycardia or ventricular fibrillation. Am J Cardiol 1993; 71: 823-826.

25. Grimm W, Hoffmann J, Menz V, Luck K, Maisch B. Programmed ventricular stimulation for arrhythmia risk prediction in patients with idiopathic dilated cardiomyopathy and nonsustained ventricular tachycardia. J Am Coll Cardiol 1998; 32: 739-745.

26. Connolly SJ, Dorian P, Roberts RS, Gent M, Bailin S, Fain ES, et al. Comparison of beta-blockers, amiodarone plus beta-blockers, or sotalol for prevention of shocks from implantable cardioverter defibrillators: The OPTIC Study: A randomized trial. JAMA 2006; 295: $165-171$. 